\title{
Making Augmented Reality Work Outdoors Requires Hybrid Tracking
}

\author{
Ronald T. Azuma, Bruce R. Hoff, Howard E. Neely III, Ronald Sarfaty, \\ Michael J. Daily \\ HRL Laboratories \\ 3011 Malibu Canyon Road; Malibu, CA 90265 \\ Office: (310) 317-\{5151, 5674, 5829, 5013, 5673\}; Fax: (310) 317-5695 \\ \{azuma, neely, hoff, rsarfaty, mjdaily\}@HRL.com \\ Gary Bishop, Vern Chi, Greg Welch \\ University of North Carolina at Chapel Hill \\ CB \#3175 Computer Science Dept.; Chapel Hill, NC 27599-3175 \\ Office: (919) 962-\{1886, 1742, 1819\}; Fax: (919) 962-1799 \\ \{gb, chi, welch\}@cs.unc.edu \\ Ulrich Neumann, Suya You \\ University of Southern California \\ Henry Salvatori 338; Los Angeles, CA 90089-0781 \\ Office: (213) 740-\{4489, 4495\}; Fax: (213) 740-7285 \\ uneumann@cs.usc.edu, suyay@graphics.usc.edu
}

Rich Nichols, Jim Cannon

Raytheon Systems Company

2175 Park Place M/S A264; El Segundo, CA 90245

Office: (310) 616-\{4999, 3401\}; Fax: (310) 616-4729

\{rwnichols, jjcannon\} @west.raytheon.com

\begin{abstract}
Developing Augmented Reality systems that work outdoors, rather than indoors in constrained environments, will open new application areas and motivate the construction of new, more general tracking approaches. Accurate tracking outdoors is difficult because we have little control over the environment and fewer resources available compared to an indoor application. This position paper examines the individual tracking technologies available and concludes that for the near term, a hybrid solution is the only viable approach. The distortion measured from an electronic compass and tilt sensor is discussed.
\end{abstract}

\subsection{Motivation}

Several prototype Augmented Reality (AR) systems have demonstrated adequate performance to meet the needs of indoor applications, ranging from 
medical visualization [State96] to aircraft manufacturing [Nash97] and even entertainment [Ohshima98]. However, few have attempted to build Augmented Reality systems that work outdoors. A group at Columbia demonstrated the "touring machine," which allows a user to view information linked to specific buildings on the Columbia campus as he walks around outside [Feiner97]. Some wearable computers, such as the CMU VuMan system, have been used for vehicle maintentance applications in outdoor settings. None of these previous works attempted to achieve accurate registration at a wide variety of outdoor locations.

Augmented Reality systems that provide accurate registration outdoors are of interest because they would make possible new application areas and could provide a natural interface for wearable computers, an area of growing interest both in academia and industry. A user walking outdoors could see spatiallylocated information directly displayed upon her view of the environment, helping her to navigate and identify features of interest. Today, a hiker in the woods needs to pull out a map, compass, and GPS receiver, convert the GPS and compass readings to her location and orientation, and then mentally align the information from the 2-D map onto what she sees in the 3-D environment around her. A personal, outdoor AR system could perform the same task automatically and display the trail path and landmark locations directly upon her view of the surrounding area, without the cognitive load. Architectural clients could see what a proposed building would look like by walking around the construction site. Soldiers could see the locations of enemies, friends, and dangerous areas like minefields that may not be readily apparent to the naked eye. Personal, outdoor AR systems would also be useful for groups of users working together. If the users are widely separated, it is difficult for them to establish common frames of reference to describe spatially-located information. An instruction telling another team member to go to the "3rd white building to the left of the red building" may be useless if the recipient sees the world from a different vantage point than the speaker. Personal AR displays provide an unambiguous method of sharing such information. Furthermore, personal outdoor AR displays may be a natural interface for wearable PC's. The standard WIMP interface does not map well onto wearable PCs, because the desktop metaphor is not appropriate for a user walking around outdoors who may not have her hands free or be able to allocate complete attention to the computer [Rhodes98]. Augmented Reality may be a better approach for certain applications.

What are the difficulties that prevent personal, outdoor AR systems from being deployed today? The ergonomic issues that face wearable PC systems apply to outdoor AR systems as well: size, weight, power, ruggedness, etc. Displays that have sufficient contrast to work in outdoor settings are required. But the biggest challenge lies in accurate tracking outdoors: determining the user's position and orientation with sufficient accuracy to avoid 
significant registration errors. This position paper focuses on the tracking problem and discusses the issues involved.

Accurate tracking indoors is hard enough; accurate tracking outdoors is even more daunting because of two main differences in the situation. First, we have less control over the environment. Second, we have fewer resources available -- power, computation, sensors, etc. These differences mean that solutions for indoor AR may not directly apply to personal outdoor AR systems. For example, several indoor AR systems have achieved accurate tracking and registration by carefully measuring the objects in a highly constrained environment, putting colored dots over those objects, and tracking the dots with a video camera (see [Azuma97] for some references). This approach violates some of the constraints for an outdoor situation. We do not have control over the outdoor environment and cannot always rely on modifying it to fit the needs of the system. For example, in a military application it is not realistic to ask soldiers, friendly or enemy, to wear large, brightly colored dots to aid our tracking system. We should not expect to measure every object in the environment beforehand. Also, this approach may require more computational resources than is practical for a single outdoor user. Such systems have used an SGI Onyx or other high-end workstation with framegrabbing capability. However, outdoor AR applications may demand less registration accuracy than many indoor applications. A doctor performing a needle biopsy requires that the virtual incision marker be accurate within a millimeter, but for a hiker walking around, perhaps even one degree of angular error is acceptable to cue the user to landmarks in the environment.

\subsection{Analysis}

If we analyze the tracking technologies available outdoors, we find that no single technology provides a complete solution. Combining several tracking technologies, or hybrid tracking, is the only feasible approach for the near term. Hybrid approaches increase system complexity and cost but often provide the most robust results. We now briefly describe the strengths and weaknesses of individual technologies, including an analysis of data taken from a sourceless orientation sensor.

- GPS: The Global Positioning System provides worldwide coverage and measures the user's 3-D position typically within 30 meters for regular GPS and about 3 meters for differential. It does not measure orientation. Differential accuracy is sufficient for viewing distant but not nearby objects; at 50 meters range a 3 meter position error results in 3.4 degrees of registration error. New carrier-phase GPS receivers claim accuracy in centimeters, but multipath problems make that difficult to achieve in many outdoor situations. GPS requires direct line-of-sight to the satellites and is commonly blocked in urban areas, canyons, etc. In military situations, GPS is easily jammed. 
- Inertial and dead reckoning: Inertial sensors are sourceless and relatively immune to environmental distrubances. Their main problem is drift; they accumulate error with time, and existing inertial sensors of the cost and weight appropriate for a single person drift too quickly to be the sole solution. Using accelerometers to track position is especially difficult due to the double integration required; this requires accelerometers with bias accuracies several orders of magnitude better than what is commonly available today.

- Active sources: Setting up active transmitters and receivers (using magnetic, optical, or ultrasonic technologies) is commonly done for indoor Virtual Environment systems but modifying the environment in this manner outdoors is not usually practical and restricts the user to the location of the active sources.

- Passive optical: Using video sensors to track the user location based upon what is visible (the sun, the stars, and the view of the surrounding environment) must play an important part. It provides the ability to do closedloop tracking approaches. Video sensors are line-of-sight and will lose lock if the view is obscured (by buildings, vegetation, etc.) Computer vision techniques are not currently robust enough to provide a complete sole solution, and unless known landmarks are used, the tracking solutions are relative rather than absolute. Video processing is also computationally intensive and not feasible in real time on most wearable PC systems.

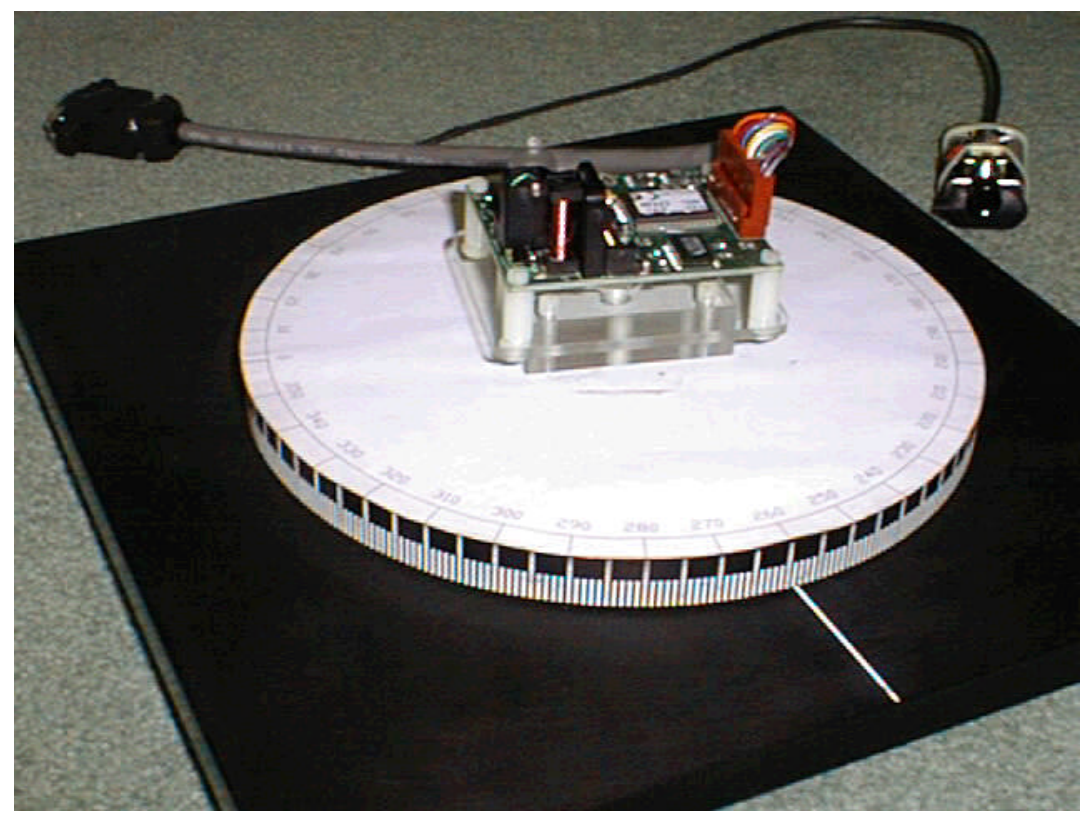

Figure 1: Non-metallic turntable for measuring compass distortion. 
- Electronic compass and tilt sensors: Many inexpensive HMD's use a sourceless orientation-only head tracker that consists of an electronic compass and two tilt sensors. The tracker is small and inexpensive and claims yaw accuracy of \pm 0.5 degrees. The compass is highly sensitive to disturbances in the ambient magnetic field. Figure 1 shows the equipment we used to measure distortions in the field. We built this mechanical turntable out of Delrin to avoid adding any sources of magnetic distortion. The measurements were taken outdoors at several different locations, far from any apparent sources of distortion. Even in such ideal environments, the compass measurements would be in error by up to 2-3 degrees, and the distortion pattern varies significantly with time and location (Figure 2). The sensor's output is also delayed in time by roughly 100 milliseconds due to the settling time of the tilt sensors, and the output is noisy and displays noticeable overshoots during motion. When near metal objects, the distortion can increase to 20-30 degrees. While this sensor may be sufficient for some games and is helpful as part of a hybrid solution, our measurements strongly suggest that by itself such a tracker is inadequate for outdoors AR.

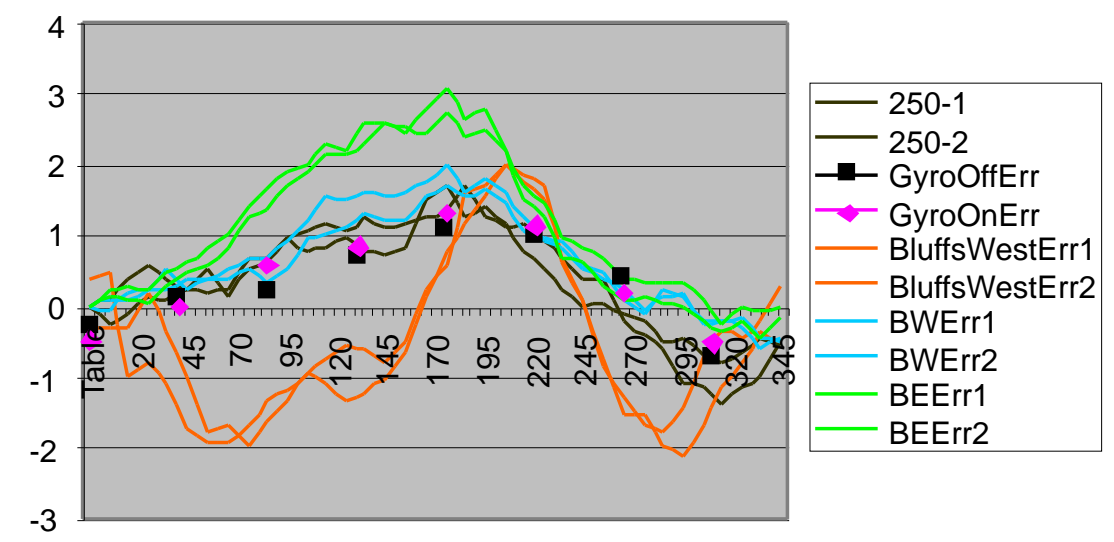

Figure 2: Distortion in electronic compass (Precision Navigation TCM2). X axis is yaw orientation of compass in degrees, $Y$ axis is the relative error in degrees from the zero degree initial orientation.

Since no single tracking technology appears adequate to provide a complete solution, the authors have formed a team collaborating to research hybrid tracking for outdoors AR, combining the technologies listed above with others yet to be developed. This work is funded by DARPA under the GRIDS project (Geospatial Registration of Information for Dismounted Soldiers). 


\section{Acknowledgements}

2013.

Work in this area is mostly funded by DARPA contract N00019-97-C-

\section{References}

Azuma97

Feiner97

Nash97

Ohshima98

Rhodes98

State96
Azuma, Ronald T. A Survey of Augmented Reality. Presence: Teleoperators and Virtual Environments 6, 4 (August 1997), 355-385.

Feiner, Steven, Blair MacIntyre, and Tobias Höllerer. A Touring Machine: Prototyping 3D Mobile Augmented Reality Systems for Exploring the Urban Environment. Proceedings of First International Symposium on Wearable Computers (Cambridge, MA, 13-14 October 1997), 74-81.

Nash, Jim. Wiring the Jet Set. Wired 5, 10

(October 1997), 128-135.

Ohshima, Toshikazu, Kiyohide Satoh, Hiroyuki

Yamamoto and Hideyuki Tamura. AR ${ }^{2}$ Hockey: A Case Study of Collaborative Augmented Reality.

Proceedings of IEEE VRAIS '98 (Atlanta, GA, 14-18 March 1998), 268-275.

Rhodes, Bradley. WIMP Interface Considered Fatal. Position paper at IEEE VRAIS '98 Workshop on Interfaces for Wearable Computers (Atlanta, GA, 15 March 1998).

State, Andrei, Mark A. Livingston, Gentaro Hirota, William F. Garrett, Mary C. Whitton, Henry Fuchs and Etta D. Pisano. Techniques for AugmentedReality Systems: Realizing Ultrasound-Guided Needle Biopsies. Proceedings of SIGGRAPH '96 (New Orleans, LA, 4-9 August 1996), 439-446. 